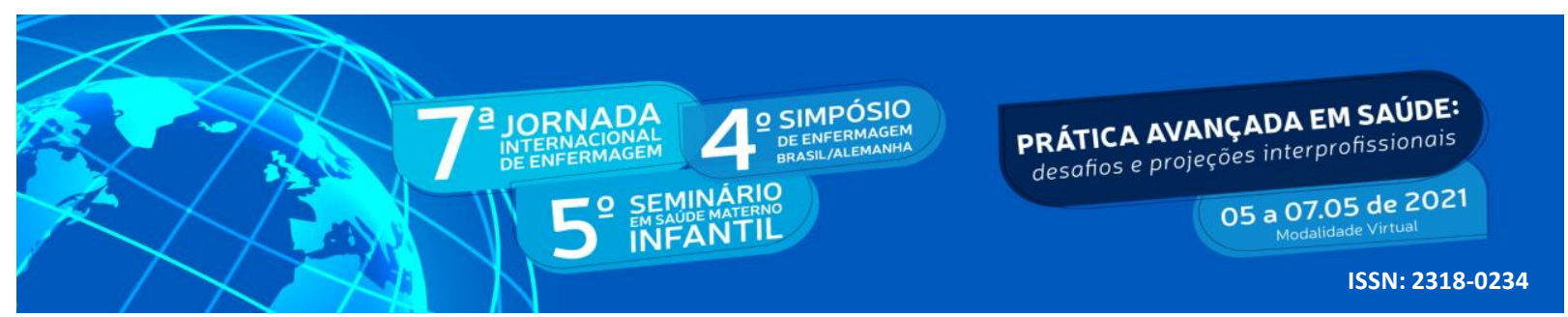

DOI: http://doi.org/10.48195/jie2021-169

\title{
ELABORAÇ̃̃O DE VÍDEO EDUCATIVO COMO FERRAMENTA DE INFORMAÇÃO AO PÚBLICO: UM RELATO DE EXPERIÊNCIA
}

\author{
Letícia Hadlich Correa de Barrosi'; Rejane Bermudes Costa Beber²; Tatiana Lins \\ Alves $^{3}$; Franceliane Jobim Benedetti ${ }^{4}$; Patrícia Pasquali Dotto ${ }^{5}$
}

\begin{abstract}
RESUMO
A deficiência de ferro é a principal causa de anemia no mundo e considerada problema de saúde pública. Acomete todos os estratos sociais, sendo mais grave nas populações de maior vulnerabilidade, principalmente em crianças menores de dois anos . O objetivo do presente trabalho é descrever o processo de criação de um "drops" (mensagem curta em formato de vídeo) com a finalidade de divulgar informações sobre prevenção da anemia ferropriva. O relato de experiência descreve o desenvolvimento do projeto, com seus desafios e aprendizados, que foi proposto na disciplina de Vigilância, Prevenção de Riscos e Agravos à Saúde Materno-Infantil do Mestrado em Saúde Materno-Infantil da UFN. O material final foi divulgado na UFN TV com o título "Drops Cuidando com Amor" e atingiu um dos objetivos da disciplina: aprimorar ações em saúde. O trabalho incentivou os participantes para realização de futuros projetos baseados nas habilidades e competências alcançadas na disciplina.
\end{abstract}

Palavras-chave: Anemia ferropriva; Prevenção; Dieta; Criança.

\begin{abstract}
Iron deficiency is the leading cause of anemia in the world and considered a public health problem. Affects all social stratum, being more severe in population with greater vulnerability, mainly in under 2 years old children. This study objective is to describe the "drops" creation process ( short message in video format) aimed to disseminate information about iron deficiency anemia. The experience report describes the project development, with its challenges and discoveries, which was proposed in a discipline Maternal-Child Health Surveillance, Risk

\footnotetext{
${ }^{1}$ Médica. Mestranda do Curso de Mestrado Profissional Saúde Materno Infantil da Universidade Franciscana, Santa Maria/RS - Brasil. leticiahcbf@gmail.com

2 Médica. Mestranda do Curso de Mestrado Profissional Saúde Materno Infantil da Universidade Franciscana, Santa Maria/RS - Brasil. rejanebermudes@gmail.com

${ }^{3}$ Médica. Mestranda do Curso de Mestrado Profissional Saúde Materno Infantil da Universidade Franciscana, Santa Maria/RS - Brasil. tatianalinspedi@gmail.com

${ }^{4}$ Nutricionista. Professora do Curso de Mestrado Profissional Saúde Materno Infantil da Universidade Franciscana, Santa Maria/RS - Brasil. franceliane.b@gmail.com

${ }^{5}$ Odontóloga. Professora do Curso de Mestrado Profissional Saúde Materno Infantil da Universidade Franciscana, Santa Maria/RS - Brasil. ppdotto@ufn.edu.br
} 


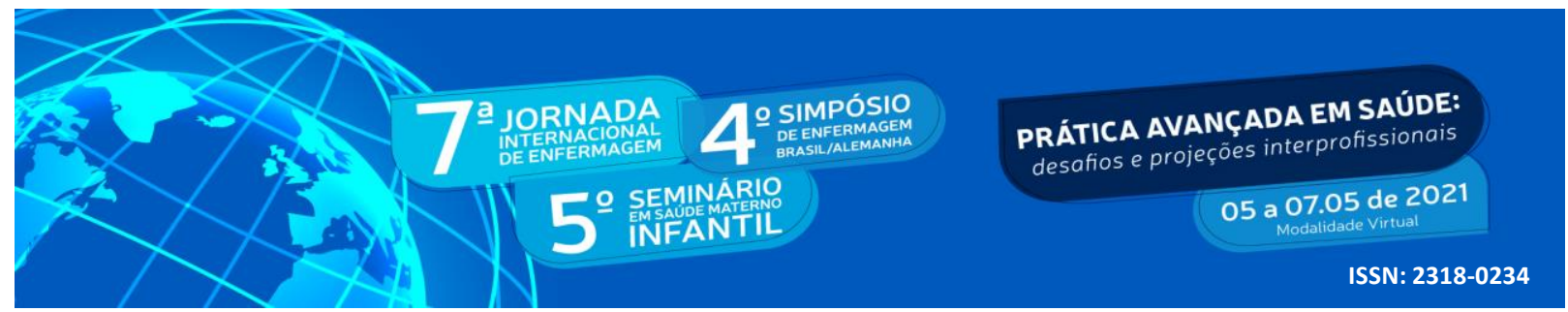

Prevention and Harm, of the Maternal and Child Health master's degree at UFN. The final material was released on UFN TV with the title "Drops Cuidando com Amor" and achieved one of the discipline objectives: improve health actions. The study encouraged participants for realization of future projects based on the skills and competencies achivied in discipline.

Key Words: Iron Deficiency Anemia; Prevention; Dietary; Child.

\section{INTRODUÇÃO}

Segundo a Organização Mundial da Saúde (OMS), o conceito de anemia configura uma condição onde a hemoglobina sérica encontra-se abaixo de valores esperados (menor que -2 DP), não sendo assim suficiente para atender as necessidades fisiológicas do organismo conforme a idade, sexo, gestação e altitude (OMS, 2015). Trata-se de uma patologia com origem multifatorial, podendo ser causada pela deficiência de ferro, deficiência de micronutrientes, perdas sanguíneas, processos patológicos e infecções, além do uso de medicações que impossibilitem ou prejudiquem o processo de absorção do ferro (OMS, 2017; CAMASCHELLA, 2015).

A deficiência de ferro é considerada a principal causa mundial de anemia, sendo responsável por $60 \%$ da totalidade de casos (KASSEMBAUM, 2016). A anemia por deficiência de ferro acomete todos os estratos sociais, podendo ser maior em populações com maior vulnerabilidade social. Acomete principalmente crianças menores de cinco anos, e particularmente aquelas menores de dois anos de idade, devido a elevada demanda de ferro que esta faixa etária exige em função do rápido crescimento e desenvolvimento, associado a pobre ingestão do mineral na alimentação complementar, principalmente $o$ ferro de alta biodisponibilidade (OMS, 2015; OMS, 2017; SUNDARARAJAN, RABE, 2021).

Ações de educação alimentar e nutricional voltadas para a prevenção da anemia ferropriva possibilitam o acesso da população às orientações sobre aleitamento materno exclusivo e prolongado e alimentação adequada, de forma a estimular o consumo de alimentos fontes de ferro e de outros que aumentam sua biodisponibilidade e absorção pelo organismo durante a introdução de alimentos complementares na dieta (SBP, 2018).

A combinação de estratégias nutricionais que possibilitem oferta e absorção de ferro adequadas é a melhor abordagem para melhorar as reservas de ferro no organismo. Carnes de gado, peixe, ave, porco, cordeiro e fígado são fontes ricas de ferro biodisponível, de origem animal. 


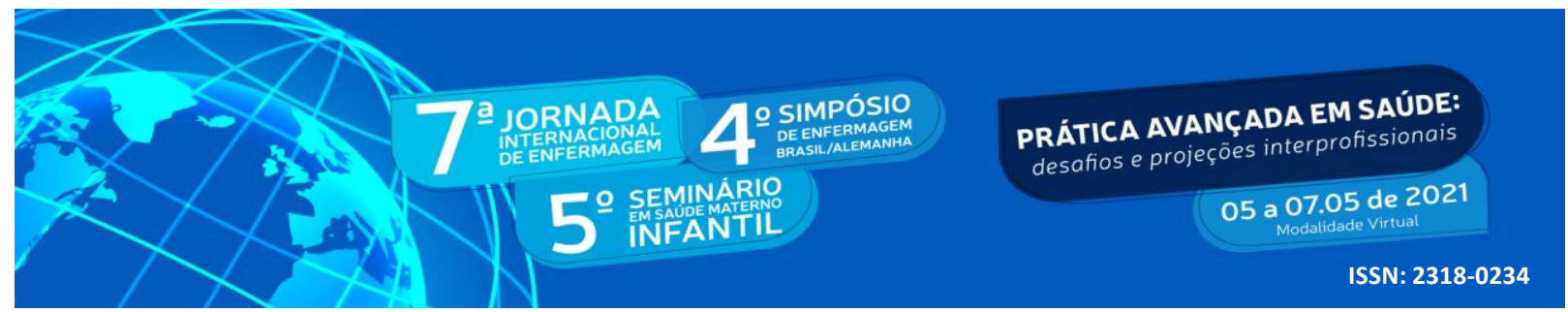

Leguminosas como feijão, ervilha, lentilha e soja; vegetais verde-escuros, como mostarda, acelga, couve, brócolis e almeirão, são fontes vegetais de ferro e necessitam ser ingeridos em combinação com alimentos ricos em vitamina $\mathrm{C}$ para melhorar a absorção do ferro contidos nestes alimentos. Além disso, adicionar alimentos fontes de ferro de origem animal à alimentos fontes de ferro de origem vegetal irá aumentar a biodisponibilidade e a absorção de ferro da refeição. Finalmente, evitar a combinação de inibidores do ferro de alimentos de origem vegetal, como chá, café e leite/derivados, com as refeições, melhora a absorção do ferro (OMS, 2017).

Está comprovado que os alimentos ricos em ferro são consumidos em quantidades insuficientes por crianças, principalmente abaixo de dois anos de idade. Por isso a importância da divulgação de orientações alimentares adequadas para prevenção de anemia ferropriva (OMS, 2017; SBP, 2018).

As orientações para prevenção de anemia podem ser vinculadas a diferentes ferramentas, entre elas o "Drops", que é um termo que designa um conteúdo, podendo ser em áudio, vídeo ou texto, geralmente curto, e que é divulgado nas mídias digitais e impressas para transmitir uma informação. Esse método é, pelo seu conteúdo simples, direto e compreensível, e devido ao seu grande alcance, principalmente em rádio e internet, muito utilizado atualmente tanto para entretenimento quanto para divulgação de notícias. Essas características permitem que os "drops" sejam elaborados com informações relevantes dirigidas para públicos específicos (DICIONÁRIO DE JORNALISMO, 2020).

Dessa forma, optou-se pela construção de um “drops”. Um vídeo curto para transmitir, através da televisão, orientações relevantes sobre a anemia ferropriva para a população.

\section{OBJETIVO}

O objetivo do presente trabalho é descrever o processo de criação de um "drops" (mensagem curta em formato de vídeo) com a finalidade de divulgar informações sobre a prevenção da anemia ferropriva.

\section{METODOLOGIA}

O presente trabalho trata-se de um relato de experiência, que descreve uma vivência profissional e cujo processo de realização contribui positivamente para proposição de diferentes 


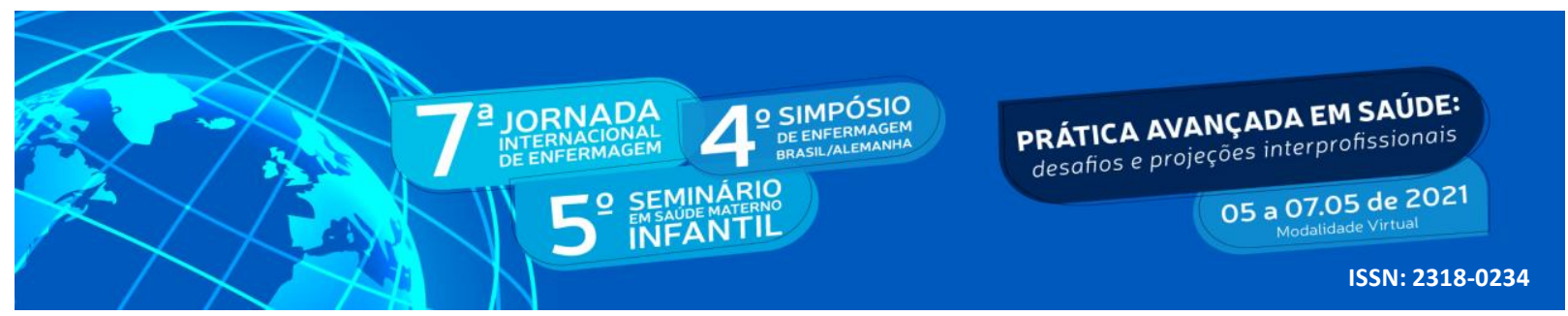

ideias de melhoria no cuidado em saúde (UFJF, 2016). Foi realizado de modo contextualizado, objetivo e baseado em conteúdo teórico.

A experiência descrita foi proposta como atividade final da disciplina de Disciplina de Vigilância, Prevenção de Riscos e Agravos à Saúde Materno-Infantil do Curso de Mestrado Profissional em Saúde Materno-Infantil da Universidade Franciscana. Trata-se de uma disciplina que tem como objetivos a melhoria do conhecimento a respeito dos conceitos em vigilância em saúde e aprofundar o conhecimento sobre a prevenção de riscos e agravos à saúde materno-infantil. O propósito da disciplina é possibilitar aos profissionais da saúde o desenvolvimento de boas práticas em saúde e ações preventivas. Além disso, destaca a importância das ações de promoção de saúde materno-infantil conforme as políticas em saúde.

O período de construção do Drops foi de vinte dias. Teve seu início na escolha do tema, logo depois realizou-se a escrita do roteiro e em uma terceira etapa realizou-se a gravação do vídeo. O mesmo foi apresentado para os demais mestrandos da disciplina e avaliado pelas docentes. O material finalizado foi veiculado na UFN TV e no YouTube da rede de televisão.

\section{DESENVOLVIMENTO}

Ao final da disciplina de Vigilância, Prevenção de Riscos e Agravos à Saúde MaternoInfantil, em outubro de 2020, os alunos foram desafiados pelas docentes da disciplina, com uma metodologia ativa e inovadora de ensino e aprendizagem: a elaboração de um "drops", o qual seria utilizado como veículo de informação e promoção em saúde. O termo "drops" é uma palavra da língua inglesa que significa gota. No jornalismo esse jargão é utilizado para nomear notícias em texto, áudio ou vídeos de curta duração que aparecem durante o intervalo de uma programação televisiva ou de rádio (DICIONÁRIO DE JORNALISMO, 2020).

Inicialmente foi mostrado pelos professores alguns exemplos de "drops" e discutido seu conceito e utilização em ações em saúde. Logo em seguida, em direção ao objetivo principal da disciplina, foi solicitado aos alunos que sugerissem temas que permeassem a promoção de saúde e prevenção de agravos, contemplados no conteúdo programático da disciplina. Os alunos foram divididos em grupos para a realização dos trabalhos e o nome do 


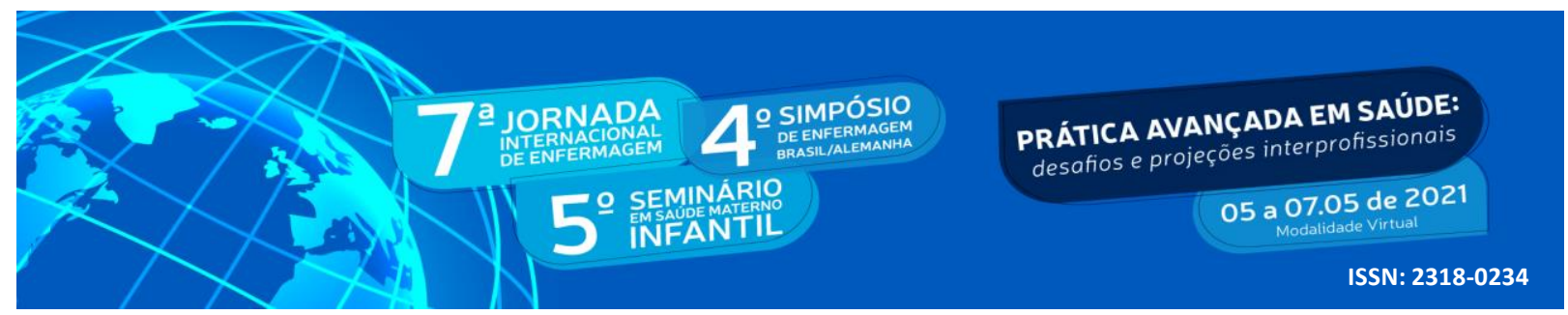

conjunto de "drops" confeccionados foi decidido em reunião da turma. O nome escolhido foi "Cuidando com Amor", devido a um dos objetivos principais da disciplina: promover saúde.

Os autores do presente trabalho escolheram como tema a anemia ferropriva na infância, devido à elevada prevalência dessa patologia. Conforme o descrito na Pesquisa Nacional de Demografia e Saúde, a prevalência da anemia ferropriva é de 20,9\% entre menores de cinco anos e de $24,1 \%$ em crianças menores de dois anos (BRASIL, 2009). Além disso, o Programa Nacional de Suplementação de Ferro coloca que para prevenir a anemia devido a deficiência de ferro necessitam-se diferentes estratégias, entre elas ações de educação nutricional e alimentar que visem uma alimentação adequada e saudável (BRASIL, 2013).

A partir do exposto acima, os autores realizaram buscas em publicações da Sociedade Brasileira de Pediatria para embasamento teórico na confecção do texto a ser utilizado no "drops". Após a leitura das evidências científicas, foi elaborado um roteiro descritivo, contendo introdução, desenvolvimento e finalização. Este roteiro passou pela revisão das docentes, com posterior devolutiva para que os alunos realizassem as adequações e passassem para a apreciação final e aprovação do texto. Após o aval positivo foi dado início a gravação do drops.

Um dos maiores desafios foi reunir informações relevantes, sintetizá-las e transcrever para uma linguagem objetiva e sem o uso de termos técnicos, para que fosse compreendida pelos diferentes públicos. Por utilizar as linguagens áudio e visual, o vídeo educativo permite uma maior retenção de informações, e com isso, uma maior facilidade na compreensão do conteúdo apresentado. Sendo assim, também pode auxiliar o indivíduo na reprodução das informações obtidas através dessa tecnologia audiovisual (MOREIRA et al., 2013).

Frente ao cenário da atual pandemia pelo COVID-19 (SARS-Cov-2), todos os protocolos e cuidados foram obedecidos a fim de se garantir a segurança dos envolvidos no processo de produção do material.

Para a gravação do "drops" optou-se por um cenário simples e que remetesse ao ambiente dos domicílios das pessoas, contendo uma mesa onde foram colocados em exposição diversos alimentos ricos em ferro. Isso para que, além de ouvir o conteúdo informativo, o público pudesse visualizar as cores dos alimentos citados._No dia 11 de outubro de 2020 realizou-se a gravação do vídeo. Foram seguidas todas as orientações realizadas pela UFN TV 


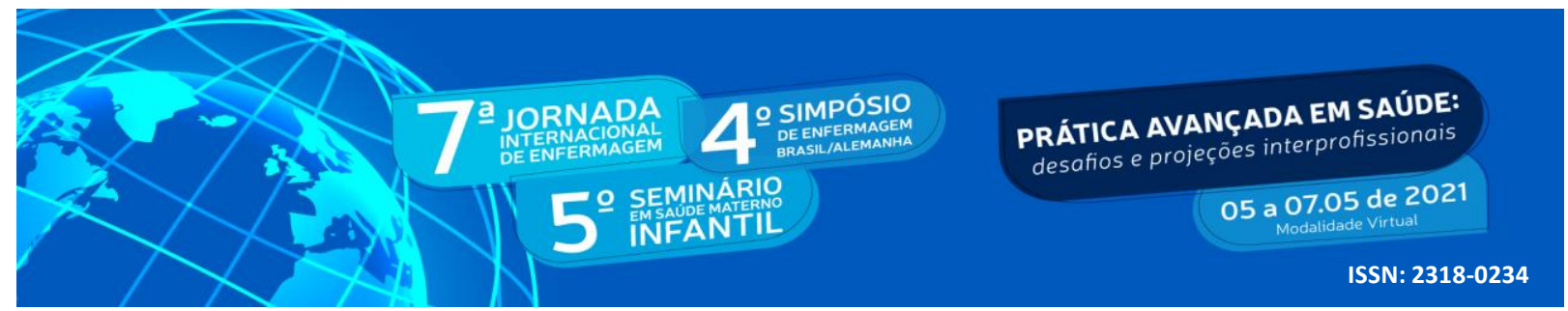

durante o processo de gravação. Apenas três tentativas foram necessárias, sendo uma delas inutilizada devido a ruídos oriundos do ambiente. Em seguida, a gravação foi encaminhada às professoras da disciplina que analisaram o conteúdo e então enviaram à equipe de produção da UFN TV, que avaliou a qualidade do material e realizou as edições necessárias.

Após a conclusão, os trabalhos foram apresentados durante as aulas, incluindo todas as gravações realizadas pelos alunos. Isso permitiu a avaliação do desempenho das equipes pelos próprios alunos e pelas professoras da disciplina. O conjunto de drops "Cuidando com Amor" passou a ser transmitido durante os intervalos da programação da UFN TV, além de ser divulgado no canal do Youtube da mesma rede de televisão.

\section{CONCLUSÃO}

O trabalho realizado mostrou que ações em saúde podem ser feitas das mais variadas formas.

Sendo a anemia ferropriva um problema de saúde pública, entende-se a importância da difusão de informações sobre medidas preventivas de forma que esse conteúdo alcance os mais diversos públicos.

Conclui-se que um "drops” em formato de vídeo com transmissão na mídia em geral possui um grande alcance para a divulgação de informações ao público, principalmente pelo fato de ser dinâmico, envolver sons e imagens, possuir curta duração e permitir a apresentação de uma linguagem simples, objetiva e clara, devendo ser considerado como importante ferramenta de ação e educação em saúde materno-infantil.

\section{REFERÊNCIAS}

BRASIL. Ministério da Saúde. Pesquisa Nacional de Demografia e Saúde da Criança e da Mulher PNDS 2006: Dimensões do Processo Reprodutivo e da Saúde da Criança. Disponível em: http://bvsms.saude.gov.br/bvs/pnds/. Acesso em: 14 abr. 2021.

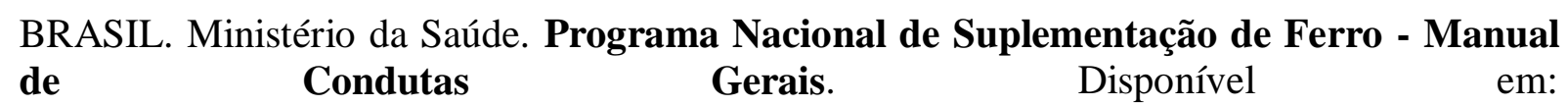
https://bvsms.saude.gov.br/bvs/publicacoes/manual_suplementacao_ferro_condutas_gerais.pd f. Acesso em: 14 abr. 2021. 


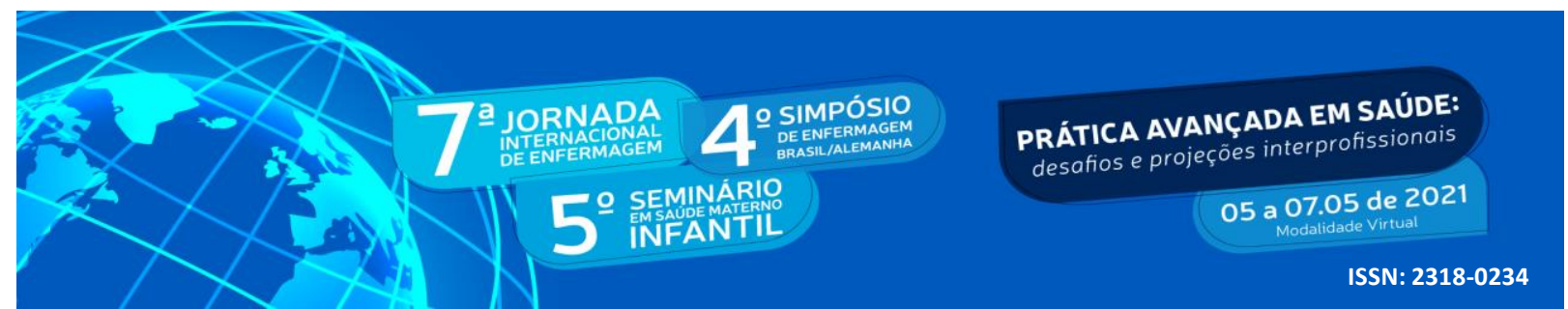

CAMASCHELLA, C. Iron-Deficiency Anemia. The New England Journal of Medicine, v. 372 , n. 19, p. 1832-1843, mai./2015.

DICIONÁRIO DE JORNALISMO. Drops. Disponível em: https://dicionariodejornalismo.blogspot.com/2020/04/drops.html. Acesso em: 2 mar. 2021. KASSEBAUM, N J. The Global Burden of Anemia. Hematology/Oncology Clinics of North America, v. 30, n. 2, p. 247-308, set./2016.

MOREIRA, C.B. et al. Construção de um Vídeo Educativo sobre Detecção Precoce do Câncer de Mama. Revista Brasileira de Cancerologia, v. 59, n. 3, p. 401-407, jun./2013.

SOCIEDADE BRASILEIRA DE PEDIATRIA. Consenso sobre Anemia Ferropriva: Mais que uma doença, uma emergência médica. Disponível em:

https://www.sbp.com.br/fileadmin/user_upload/21019f-

Diretrizes_Consenso_sobre_anemia_ferropriva-ok.pdf. Acesso em: 2 mar. 2021.

SUNDARARAJAN, S.; RABE, H. Prevention of iron deficiency anemia in infants and toddlers. Pediatric Research, v. 89, n. 1, p. 63-73, 2021.

UNIVERSIDADE FEDERAL DE JUIZ DE FORA. Instrutivo para elaboração de relato de experiência. Departamento de Nutrição. Disponível em:

https://www.ufjf.br/nutricaogv/files/2016/03/Orienta\%C3\%A7\%C3\%B5es-

Elabora\%C3\%A7\%C3\%A3o-de-Relato-de-Experi\%C3\%AAncia.pdf. Acesso em: 2 mar.

2021.

WORLD HEALTH ORGANIZATION. Nutritional anaemias: tools for effective prevention and control. Geneva: World Health Organization. Disponível em: www.who.int/publications/i/item/9789241513067. Acesso em: 16 mar. 2021.

WORLD HEALTH ORGANIZATION. The global prevalence of anaemia in 2011. Disponível em:

https://www.who.int/nutrition/publications/micronutrients/global_prevalence_anaemia_2011/ en/. Acesso em: 2 mar. 2021. 